\title{
How does intentionality of encoding affect memory for episodic information?
}

\author{
Michael Craig, ${ }^{1,2}$ Karla Butterworth, ${ }^{2}$ Jonna Nilsson, ${ }^{2,3}$ Colin J. Hamilton, ${ }^{4}$ \\ Peter Gallagher, ${ }^{2}$ and Tom V. Smulders ${ }^{2}$ \\ ${ }^{1}$ Department of Psychology, School of Life Sciences, Heriot-Watt University, Edinburgh, EH14 4AS, United Kingdom; ${ }^{2}$ Institute of \\ Neuroscience, Newcastle University, The Henry Wellcome Building for Neuroecology, Framlington Place, Newcastle upon Tyne, NE2 \\ $4 \mathrm{HH}$, United Kingdom; ${ }^{3}$ Aging Research Center, Karolinska Institutet, Stockholm, SE-113 30, Sweden; ${ }^{4}$ Department of Psychology, \\ Northumbria University, Northumberland Building, Newcastle upon Tyne, NE1 8ST, United Kingdom
}

\begin{abstract}
Episodic memory enables the detailed and vivid recall of past events, including target and wider contextual information. In this paper, we investigated whether/how encoding intentionality affects the retention of target and contextual episodic information from a novel experience. Healthy adults performed (1) a What-Where-When (WWW) episodic memory task involving the hiding and delayed recall of a number of items (what) in different locations (where) in temporally distinct sessions (when) and (2) unexpected tests probing memory for wider contextual information from the WWW task. Critically, some participants were informed that memory for WWW information would be subsequently probed (intentional group), while this came as a surprise for others (incidental group). The probing of contextual information came as a surprise for all participants. Participants also performed several measures of episodic and nonepisodic cognition from which common episodic and nonepisodic factors were extracted. Memory for target (WWW) and contextual information was superior in the intentional group compared with the incidental group. Memory for target and contextual information was unrelated to factors of nonepisodic cognition, irrespective of encoding intentionality. In addition, memory for target information was unrelated to factors of episodic cognition. However, memory for wider contextual information was related to some factors of episodic cognition, and these relationships differed between the intentional and incidental groups. Our results lead us to propose the hypothesis that intentional encoding of episodic information increases the coherence of the representation of the context in which the episode took place. This hypothesis remains to be tested.
\end{abstract}

Many everyday activities critically depend on our ability to vividly recall experiences from the past. This is a core function of the episodic memory system (Tulving 1972), which enables the recall of specific past events and wider contextual information, i.e., what happened, and where and when did it occur (Tulving 1985). Importantly, it is possible to recall past events at a later point in time, despite (1) incidentally encoding the initial event (Neill et al. 1990; Zentall et al. 2008; Holland and Smulders 2011) and (2) subsequent retrieval often being unexpected, e.g., cued by sensory impressions (Berntsen et al. 2013). To support such retrieval, the episodic memory system recruits a number of underlying cognitive functions, including verbal, visual, and spatial memory functions, as well as internal imagery and the mental manipulation of space (Grady et al. 1998; Eichenbaum 2004; Meulenbroek et al. 2004; Schacter et al. 2007; Addis et al. 2009; Plancher et al. 2010; Pause et al. 2013). Together, these functions provide the complex framework for the detailed recall and vivid reexperiencing of the past, enabling the retrieval of both target and wider contextual information.

The episodic memory system has been implicated in the performance of a number of different memory measures, from the "gold standard" retention of new verbal information, e.g., a list of words (e.g., Gavett and Horwitz 2012; Pause et al. 2013), to tests of visual memory, mental imagery, and spatial memory/navigation (Burgess et al. 2002; Hassabis et al. 2007; Pengas et al. 2010; Plancher et al. 2012; Schacter et al. 2012). Many of these tests

Corresponding author: tom.smulders@ncl.ac.uk

Article is online at http://www.learnmem.org/cgi/doi/10.1101/Im.041491. 115 . are considered to be robust and reliable measures of episodic memory ability (Plancher et al. 2008, 2012), with the assumption that similar underlying functions are recruited during the performance of each measure. If so, performance of these measures would be expected to correlate strongly. However, a growing body of research reveals difficulty in establishing such inter-task relationships, suggested to be the result of different "putative" episodic memory tests relying on the recruitment of different underlying cognitive functions (e.g., Holland and Smulders 2011; Cheke and Clayton 2013, 2015; Pause et al. 2013). Furthermore, it remains poorly understood whether the specific cognitive function(s) recruited during the performance of such "putative" tests accurately represent those recruited in everyday episodic memory use.

Pause et al. (2013) proposed that for an episodic memory test to be ecologically valid, it must: (1) be conducted in a laboratory environment, (2) not include explicit instructions to memorize to-be-retained information, (3) include a state of emotional valence, (4) be a one-trial learning event, (5) include the retention of what, where and when information, (6) include an unexpected delayed memory test, and (7) include a retention interval between learning and test of at least $60 \mathrm{~min}$ to allow for memory consolidation processes to occur. Interestingly, many

\footnotetext{
C 2016 Craig et al. This article is distributed exclusively by Cold Spring Harbor Laboratory Press for the first 12 months after the full-issue publication date (see http://learnmem.cshlp.org/site/misc/terms.xhtml). After 12 months, it is available under a Creative Commons License (AttributionNonCommercial 4.0 International), as described at http://creativecommons. org/licenses/by-nc/4.0/.
} 
of the aforementioned "putative" episodic memory measures fail to meet some/most of these criteria. Some other tests, including autobiographical memory measures, do fulfill the criteria, but such measures provide challenges in the verification of the accuracy of recalled memories. However, one task that fulfills the criteria of Pause et al. (2013) and provides directly measurable outcomes where accuracy can be reliably verified, is the recently developed What-Where-When $(W W W)$ memory task.

Based on naturalistic studies investigating episodic-like memory in nonhuman animals (Clayton and Dickinson 1998; Babb and Crystal 2005; Zinkivskay et al. 2009; Inostroza et al. 2013), the human analogue of the $W W W$ memory task examines the long-term retention of item-location-session (what-wherewhen) information pertaining to a recent experience (Holland and Smulders 2011; Mazurek et al. 2015). Using a version of the human $W W W$ task, Holland and Smulders (2011) found that manipulating the intentionality of encoding (intentional vs. incidental) during the initial experience enhanced delayed memory of target $W W W$ and wider contextual episodic information. However, it remained unclear (1) exactly how encoding intentionality affected memory for target and contextual information and (2) whether encoding intentionality affected the underlying cognitive functions recruited when recalling such information. Thus, we report a new study that expands on the findings of Holland and Smulders (2011) by examining whether memory for target and contextual information from the ecological $W W W$ task relates to common factors of cognition extracted from a set of standard measures of episodic and nonepisodic cognition, and whether intentionality of encoding affects these relationships.

Forty-nine adults performed (1) a What-Where-When $(W W W)$ episodic memory task involving the hiding and delayed recall of a number of items (two types of coins; what) in different locations (where) on temporally distinct occasions (when) and (2) unexpected tests probing memory for contextual information from the $W W W$ task (Unexpected Context Questions-UCQs; Words in the Room-WintR). Critically, some participants were informed that memory for $W W W$ information would be subsequently probed (intentional group), while this came as a surprise for others (incidental group). The probing of contextual information came as a surprise for all participants. Participants also performed several standard measures of episodic and nonepisodic cognition, from which common factors were extracted via Principal Component Analyses. We examined how these factors relate to the retention of target $(W W W)$ and contextual (UCQs, WintR) information and how intentionality of encoding affects such relationships. We predicted that (1) intentional encoding would result in superior retention of target and contextual information, (2) if the $W W W$ task, UCQs and WintR measures probe episodic memory functions, relationships would be observed between these measures and the factors derived from the standard episodic tests, but not the nonepisodic tests, and (3) if intentionality of encoding affects the retention of target and contextual episodic information by modulating episodic memory functions, different relationships would be observed between factors of episodic cognition and memory measures ( $W W W$, UCQs and WintR tests) in the intentional and incidental groups. (all $P<0.05$ ).

\section{Results}

\section{Effect of intentionality of encoding on the retention of target and contextual information}

We first examined whether intentionality of encoding affected the retention of target item-location-session $(W W W)$ and contextual information (UCQs, WintR recall and WintR recognition, see Methods for test details) from the $W W W$ experience. A repeated measures ANOVA revealed a significant main effect of encoding group (intentional vs. incidental) on performance across the $W W W$ test, UCQs, WintR recall, and WintR recognition measures (all expressed as Arcsin Square Root transformed proportion correct answers; $F_{(1,47)}=44.806, P<0.001, \eta \rho^{2}=0.488$ ), due to participants in the intentional group demonstrating superior retention to those in the incidental group (all measures: $P<0.05$; see Fig. 1). A significant main effect of memory measure was also observed $\left(F_{(3,141)}=396.461, P<0.001, \eta \rho^{2}=0.894\right)$, due to significant differences in performance between all four ( $W W W$, UCQs, WintR recall and WintR recognition) measures (all pairwise comparisons: $P<0.001)$. There was no significant interaction between memory measure and encoding group $\left(F_{(3,141)}=\right.$ 3.062, $P<0.053, \eta \rho^{2}=0.061$ ), suggesting that the effect of intentionality was similar across all memory measures.

\section{Post-experimental reports}

To examine whether participants used an episodic-like method of recall when retrieving target $W W W$ information, participants were post-experimentally asked to give a detailed account of how they recalled $W W W$ information. The majority of participants reported using an episodic-like means of recall (43/49 participants, $87.75 \%)$, which did not differ between groups (intentional: $21 / 25$ participants, $84.00 \%$; incidental: $22 / 24$ participants, $\left.91.67 \% ; \chi^{2}=0.067, P=0.413\right)$. In-depth post-hoc examination of participants' episodic-like responses revealed two common themes, solving the $W W W$ task via: (1) mentally reexperiencing the $W W W$ experience, e.g., "I recalled and visualised the pattern of my movements and could remember placing coins in certain locations around the room" (participant 37-intentional group) or (2) recalling/recognizing objects within the $W W W$ environment which led to the retrieval of specific memories for

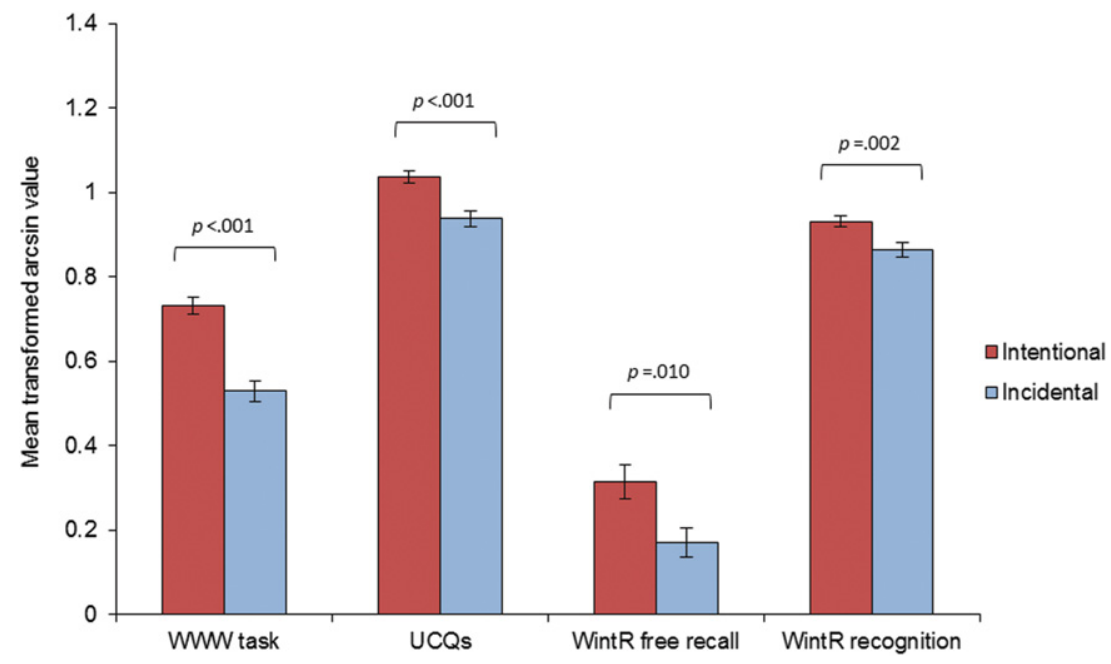

Figure 1. Mean arcsine square root transformed values for the intentional and incidental groups in the WWW task, UCQs, WintR recall, and WintR recognition tests. Error bars show the standard error of the mean. Participants in the intentional group performed significantly better across all measures 
$W W W$ information, e.g., "Some objects stood out and felt familiar, leading to the recall of memory for the coin and time of hiding" (participant 32-incidental group). In the intentional group, $15 / 21$ participants $(71.43 \%)$ reported mentally reexperiencing the $W W W$ experience, while the remaining $6 / 21$ participants $(28.57 \%)$ reported recalling/recognizing objects from the $W W W$ environment. In the incidental group, $12 / 22(54.55 \%)$ reported mentally reexperiencing the $W W W$ experience, while the remaining 10/22 participants (45.45\%) reported recalling/recognizing objects from the $W W W$ environment. This did not differ significantly between the two groups $(\chi 2=1.311, P=0.252)$. In sum, participants' post-experimental reports suggest that an episodic-like method of recall was used in both groups when retrieving $W W W$ task information.

\section{Intertask relationships among target and context memory measures}

To examine whether performances in the $W W W$ task, UCQs, WintR recall, and WintR recognition measures were related to one another, we conducted a series of Pearson correlation analyses for the intentional and incidental groups (see Table 1). For the intentional group, a significant positive correlation was found between performance in the WintR recall test and UCQs. For the incidental group, a significant positive correlation was observed between performance in the $W W W$ task and UCQs. In the incidental group, a significant correlation was also observed between the WintR recall and WintR recognition tests. No other significant correlations were observed (see Table 1). Thus, only for participants who encoded the material incidentally was there an association between memory for item-location-session information and memory for contextual information. Given the exploratory nature of these analyses, no corrections were made for multiple testing.

To explore whether Pearson correlations differed between groups, we transformed all Pearson correlation $(r)$ values via a Fisher $r$-to- $z$ transformation to normalize the data. There was a significant effect of encoding condition on the $W W W$ task-UCQs correlation $(z=-2.01, P=0.044)$, where a significant positive correlation was observed in the incidental group, and no correlation was observed in the intentional group (see Table 1). No other significant between-group differences were observed (all $P>0.05)$.

\section{PCA analysis of the episodic cognitive measures}

All participants completed a broader battery of neuropsychological tests, with a focus on measures that tap into functions in common with episodic memory tests. These measures included a verbal (wordlist) memory test, visual imagery test, mental rotation task (MRT), Northumberland gallery task (a test of egocentric and allocentric spatial memory), and an object location memory test comprising a number of submeasures: object memory $(\mathrm{OM})$, object location binding (OLB), positional object memory (POM), and combined object memory (COM) (see Methods for full test details). Given (1) that it was our aim to examine whether/how memory for target $(W W W)$ and wider contextual information related to episodic (and nonepisodic) cognitive functions and (2) performance in putative episodic memory tasks may depend on a number of different underlying cognitive functions, we conducted a principal component analysis (PCA) to reduce the data from the nine measures of episodic cognition to a smaller number of variables that should represent specific cognitive functions underlying episodic memory. An oblique Promax rotation was used, which is the recommended method when inter-component correlations are likely (Abdi 2003; Costello and Osborne 2005; Brown 2009). Unlike the $W W W$ task and measures of contextual information, there was no experimental manipulation of encoding condition (i.e., intentional vs. incidental) in these standardized measures. Data for all measures was in the same direction (or converted so that this was the case), where higher scores represented superior performance. An examination of the Kaiser-Meyer-Olkin measure of sampling adequacy suggested that the sample was factorable $(\mathrm{KMO}=0.541, P=0.001)$. Table 2 shows the rotated component matrix.

Four items loaded onto Factor 1. Table 2 indicates that what these four items may have in common is the verbal memorization of presented information (verbal memory and POM, COM, and OLB; the latter three do not require the memorization of verbal information, but verbal strategies are very likely to be used), so Factor 1 was labeled as "verbal memorization." A second factor contained three items, which have internal imagery of objects in common (visual imagery, OM, and COM), so Factor 2 was labeled as "internal imagery." The third factor also contained three items. These had the ability to mentally manipulate space in common (MRT, OLB, and NGTa), so Factor 3 was labeled as "mental manipulation of space." Finally, the fourth factor contained two items which have visual-spatial memory in common (NGTe and NGTa), so Factor 4 was labeled as "visual-spatial memory." Verbal memory was found to load negatively on to Factor 4.

\section{Predicting memory for target and contextual information from the episodic cognition factors}

To examine (1) whether memory for target $W W W$ and wider contextual information were related to the factors of episodic cognition derived from our PCA and (2) whether intentionality of encoding affected these relationships, we conducted a series of univariate ANCOVAs in which the $W W W$ task, UCQs, WintR recall, and WintR recognition measures were individually used as the dependent variable; condition (i.e., intentional vs. incidental encoding) was used as a fixed factor, and the four episodic-like PCA factors were used as covariates. Models included interactions between group and each episodic-like PCA factor. A modelsimplification approach (Crawley 2014) was adopted where the most nonsignificant interaction was removed. If the model did not produce any significant findings, the main effect associated with the excluded interaction was also removed from the model. The most nonsignificant remaining interaction was then excluded, followed by its associated main effect. This process continued

Table 1. Correlations between performances in the $W W W$ task, UCQs, WintR recall, and WintR recognition measures

\begin{tabular}{llcccrc}
\hline WWW-UCQs & $\begin{array}{c}\text { WWW- } \\
\text { WintR recall }\end{array}$ & $\begin{array}{c}\text { WWW- } \\
\text { WintR recog }\end{array}$ & $\begin{array}{c}\text { UCQs- } \\
\text { WintR recall }\end{array}$ & $\begin{array}{c}\text { UCQs- } \\
\text { WintR recog }\end{array}$ & $\begin{array}{c}\text { WintR recall- } \\
\text { WintR recog }\end{array}$ \\
\hline Intentional & $r=-0.039$, & $r=0.307$, & $r=0.038$, & $\boldsymbol{r}=\mathbf{0 . 5 8 5}$ & $r=0.240$, & $r=-0.012$, \\
Incidental & $P=0.855$ & $P=0.135$ & $P=0.858$ & $\mathbf{P}=\mathbf{0 . 0 0 2}$ & $P=0.858$ & $P=0.955$ \\
& $\boldsymbol{r}=\mathbf{0 . 5 1 9}$, & $r=0.155$, & $r=-0.141$, & $r=0.078$, & $r=-0.096$, & $\boldsymbol{r}=\mathbf{0 . 4 2 3 ,}$ \\
& $\mathbf{P}=\mathbf{0 . 0 0 9}$ & $P=0.469$ & $P=0.510$ & $P=0.719$ & $P=0.654$ & $\mathbf{P}=\mathbf{0 . 0 3 9}$ \\
\hline
\end{tabular}

Pearson correlation coefficients $(r)$ and significance $(P)$ values are shown. Correlations that reached significance $(P<0.05)$ are shown in bold. 
Table 2. Rotated component loadings for nine episodic test measures: positional object memory (POM), combined object memory (COM), visual imagery task, object memory (OM), mental rotation test (MRT), object location binding (OLB), Northumberland gallery task-egocentric trials (NGTe), Northumberland gallery task-allocentric trials (NGTa), verbal memory test

\begin{tabular}{lcrrr}
\hline Component & $\mathbf{1}$ & $\mathbf{2}$ & \multicolumn{1}{c}{$\mathbf{3}$} & \multicolumn{1}{c}{$\mathbf{4}$} \\
\hline POM & $\mathbf{0 . 8 6 6}$ & 0.272 & -0.177 & 0.086 \\
COM & $\mathbf{0 . 6 9 1}$ & $\mathbf{0 . 5 8 3}$ & 0.062 & 0.293 \\
Visual imagery & 0.222 & $\mathbf{0 . 8 1 9}$ & 0.166 & 0.078 \\
OM & 0.209 & $\mathbf{0 . 7 5 3}$ & -0.254 & -0.252 \\
MRT & -0.067 & 0.036 & $\mathbf{0 . 8 2 1}$ & -0.039 \\
OLB & $\mathbf{0 . 5 6 2}$ & -0.112 & $\mathbf{0 . 4 8 1}$ & -0.042 \\
NGTe & 0.085 & 0.014 & -0.130 & $\mathbf{0 . 7 0 8}$ \\
NGTa & 0.045 & -0.134 & $\mathbf{0 . 6 1 8}$ & $\mathbf{0 . 6 5 4}$ \\
Verbal memory & $\mathbf{0 . 5 6 2}$ & 0.228 & -0.114 & $-\mathbf{0 . 5 1 5}$ \\
Eigenvalues & 2.262 & 1.524 & 1.237 & 1.104 \\
Percentage of total variance & 25.137 & 16.931 & 13.750 & 12.267 \\
Number of test measures & 4 & 3 & 3 & 3 \\
\hline
\end{tabular}

A scree plot yielded a four-factor solution. Loadings $>0.40$ are shown in bold.

in a stepwise manner until significant findings were observed or all main effects and interactions were excluded from the model. This was done in order to examine whether, and to what degree, the factors of episodic cognition could account for the variance related to intentionality of encoding in the $W W W$ task and related measures.

For the $W W W$ task, following the removal of the most nonsignificant interaction (group*verbal memorization, $P=0.623$ ) and its corresponding main effect covariate (verbal memorization, $P=0.918)$, the main effect of group remained $\left(F_{(1,40)}=7.187\right.$, $\left.P<0.001, \eta \rho^{2}=0.557\right)$. No significant main effects of the remaining three factors of episodic cognition were observed (all $P>0.204$ ), nor were there any significant interactions (all $P>0.05)$. Therefore, none of the four episodic factors predicted performance in our target memory task, for either group.

For the UCQs, following the removal of the most nonsignificant interaction (group*verbal memorization, $P=0.647$ ) and its corresponding main effect covariate (verbal memorization, $P=0.666)$, the main effect of group remained $\left(F_{(1,40)}=14.538\right.$, $\left.P<0.001, \eta \rho^{2}=0.267\right)$. No significant main effects of the remaining three factors of episodic cognition were found (all $P>$ 0.212). However, significant interactions were observed between
(1) group and mental manipulation of space $\left(F_{(1,40)}=4.477\right.$, $\left.P=0.041, \eta \rho^{2}=0.101\right)$ and (2) group and visual-spatial memory $\left(F_{(1,40)}=5.119, P=0.029, \eta \rho^{2}=0.113\right)$. No significant interaction was observed between group and internal imagery $\left(F_{(1,40)}=\right.$ $\left.2.201, P=0.146, \eta \rho^{2}=0.052\right)$. Post hoc Pearson correlation analyses were conducted to investigate the basis of the significant interactions between (1) group and mental manipulation of space and (2) group and visual-spatial memory. These analyses revealed a significant positive relationship between performance in the UCQs and factor mental manipulation of space in the intentional group ( $r=0.440, P=0.031$, see Fig. 2$)$, but not the incidental group $(r=0.023, P=0.915)$. There was no significant correlation between performance in the UCQs and visual-spatial memory in the intentional $(r=-0.153, P=0.476)$ or incidental $(r=0.358$, $P=0.086)$ groups, but the significant interaction between visual-spatial memory and group can be accounted for by data for the two groups trending in different directions, as shown in Fig. 2.

For the WintR recall test, following the removal of the most nonsignificant interaction (group*visual-spatial memory, $P=0.959)$, the main effect of group remained $\left(F_{(1,40)}=4.943\right.$, $\left.P=0.032, \eta \rho^{2}=0.110\right)$. A significant main effect of visual-spatial memory was also observed $\left(F_{(1,39)}=4.793, P=0.035, \eta \rho^{2}=0.109\right)$. To investigate this significant main effect further we conducted a Pearson's correlation analysis between WintR recall test performance and factor visual-spatial memory, which revealed a significant positive correlation across both groups $(r=0.323, P=0.025$; see Fig. 3 for correlations for individual groups). In addition, a significant interaction between verbal memorization and group was observed $\left(F_{(1,39)}=5.260, P=0.027, \eta \rho^{2}=0.119\right)$. Pearson correlation analyses for each group revealed that the trends of the data were in opposite directions (see Fig. 3), where a positive trend was found in the intentional group $(r=0.215, P=0.314)$ and a negative trend in the incidental group $(r=-0.301, P=0.153)$.

For the WintR recognition measure, no significant main effects of the four factors of episodic cognition (all $P>0.198$ ) or significant interactions between any of the episodic factors and group (all $P>0.147$ ) were observed. Moreover, the removal of the most nonsignificant interactions in a stepwise fashion did not result in any significant interactions or main effects.

\section{PCA analysis of the nonepisodic cognitive measures}

Similar to our investigation of whether memory for target $W W W$ and contextual information was related to factors of episodic
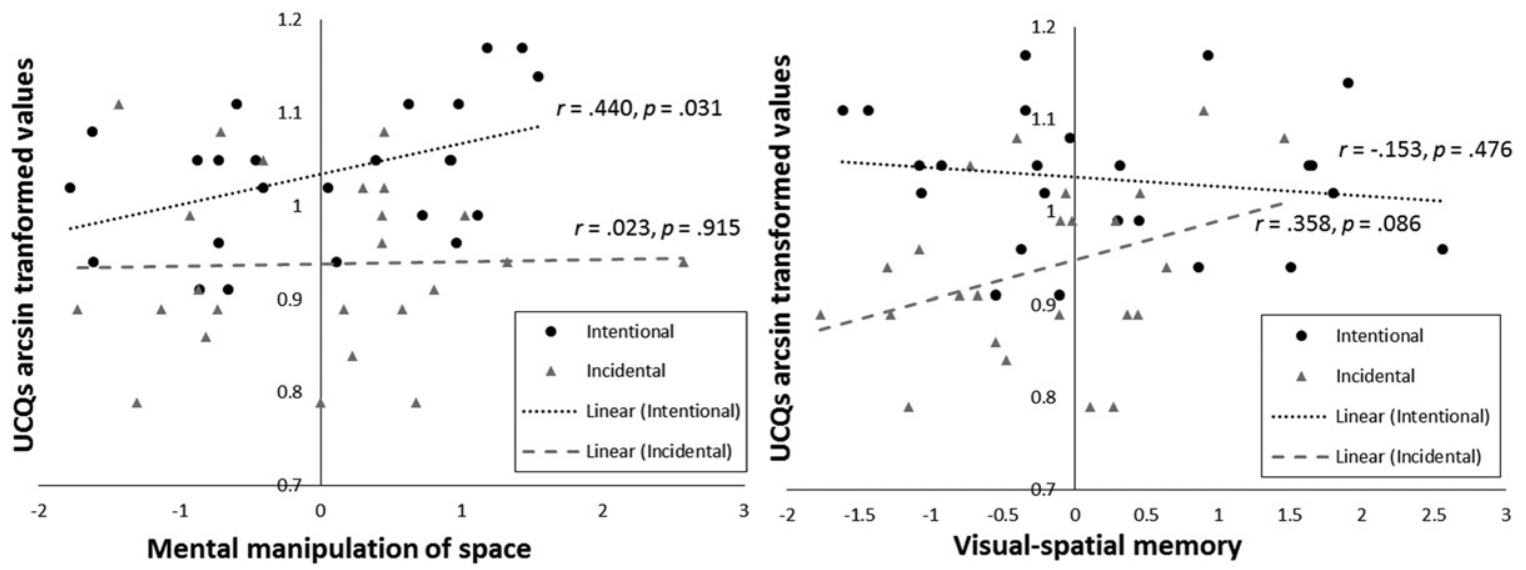

Figure 2. Interaction plots for UCQs test. Left: Scatterplot showing data trends that accounted for the significant interaction between group and mental manipulation of space. A significant correlation was observed in the intentional group. No significant correlation was observed in the incidental group. Right: Scatterplot showing data trending in different directions in the intentional and incidental groups, which accounted for the significant interaction between group and visual-spatial memory. Pearson correlation coefficients $(r)$ and significance $(P)$ values are shown. 

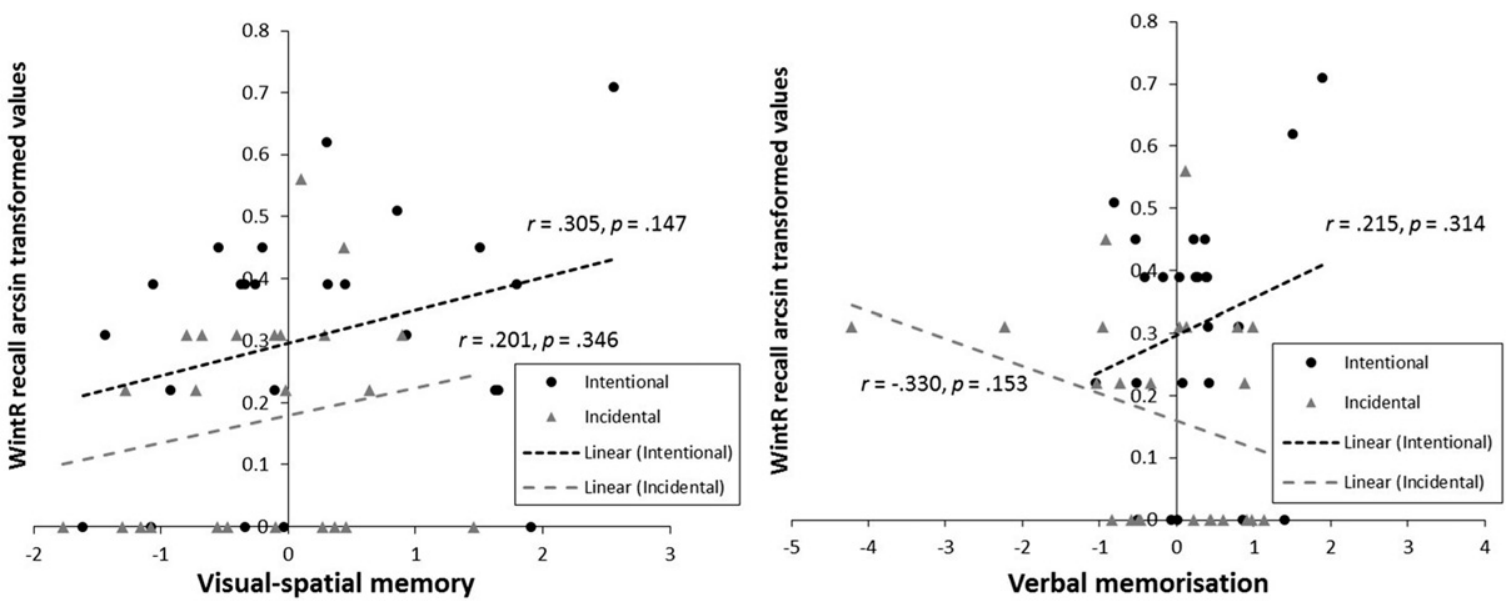

Figure 3. Interaction plots for WintR recall test. Left: Scatterplot showing positive correlations between performances in the WintR recall test and visualspatial memory in both groups (i.e., significant main effect, no significant encoding group*factor interaction). Right: Scatterplot showing data trending in different directions in the intentional and incidental groups, which accounted for the significant interaction between group and verbal memorization. Pearson correlation coefficients $(r)$ and significance $(P)$ values are shown.

cognition, participants performed a number of nonepisodic cognitive tests. These included CANTAB spatial span test, Newcastle spatial working memory (NSWM) test, visual patterns test (VPT), verbal fluency (FAS) test, Forward digit span, and Reverse digit span (see Methods section for full test details). To reduce the data from these nonepisodic memory measures to a smaller number of variables, we again conducted a PCA with an oblique Promax rotation. Data for all measures were in the same direction (or converted so that this was the case), where higher scores reflected superior performance. Like for the episodic cognition tests (and unlike the $W W W$ task), there was no between-group manipulation of encoding (i.e., intentional vs. incidental) in these tests. An examination of the Kaiser-Meyer-Olkin measure of sampling adequacy suggested that the sample was factorable $(\mathrm{KMO}=0.646$, $P<0.001)$. Table 3 shows the rotated component matrix.

Four items loaded onto Factor 1. Table 3 shows that these items related to visual-spatial working memory (CANTAB Spatial span, NSWM between errors, NSWM within errors, and VPT), so Factor 1 was labeled as "visual-spatial working memory." Three items load onto a second factor relating to the verbal working memory (verbal fluency, forward and backward digit span), so Factor 2 was labeled as "verbal working memory."

\section{Predicting target and context information from the nonepisodic cognition factors}

Univariate ANCOVAs were performed in which the $W W W$ task, UCQs, WintR recall, and WintR recognition measures were used as the dependent variable; group was used as a fixed factor, and the two nonepisodic PCA factors as covariates. Similar to the factors derived from experimental tests of episodic memory functions, a model-simplification approach (Crawley 2014) was adopted (see earlier section). For the $W W W$ task, UCQs, WintR recall, and WintR recognition measures, no significant main effects of the two nonepisodic factors (all $P>0.242$ ), nor interactions between the factors and group (all $P>0.128$ ), were observed. These results did not change following model simplification.

\section{Discussion}

In this study, we probed the effects of encoding intentionality on the long-term retention of target (i.e., item-location-session information, as tested via the $W W W$ task) and wider contextual information (i.e., memory for environmental stimuli, as tested via the UCQs and WintR recall and WintR recognition tests). Critically, some participants were informed that memory for target information would be probed subsequently (intentional group), while this came as a surprise for others (incidental group). The probing of contextual information came as a surprise for all participants. Participants also performed several standard measures of episodic/nonepisodic cognition from which common episodic and nonepisodic factors were extracted. We subsequently examined (1) whether/how these factors of episodic and nonepisodic cognition related to memory for target and contextual information and (2) whether intentionality of encoding modulated such relationships. Intentional encoding resulted in superior retention of target and contextual information. No relationships were found between memory measures and factors derived from standard nonepisodic memory measures. However, memory for contextual, but not target, information was related to factors derived from standard episodic memory measures. Importantly, these relationships differed between the intentional and incidental encoding groups. We discuss each of these findings in turn.

Table 3. Rotated component loadings for seven nonepisodic test measures: CANTAB Spatial span test, Newcastle spatial working memory test-between errors (NSWM between error), Newcastle spatial working memory test-within errors (NSWM within error), visual patterns test (VPT), verbal fluency test, forward digit span, and reverse digit span

\begin{tabular}{lcc}
\hline Component & $\mathbf{1}$ & $\mathbf{2}$ \\
\hline CANTAB spatial span & $\mathbf{0 . 8 4 0}$ & 0.109 \\
NSWM between error & $\mathbf{0 . 7 8 9}$ & 0.109 \\
NSWM within error & $\mathbf{0 . 6 7 1}$ & 0.228 \\
VPT & $\mathbf{0 . 6 8 7}$ & 0.242 \\
Verbal fluency & 0.372 & $\mathbf{0 . 5 0 8}$ \\
Forward digit span & 0.034 & $\mathbf{0 . 8 3 7}$ \\
Reverse digit span & -0.209 & $\mathbf{0 . 9 0 3}$ \\
Eigenvalues & 2.460 & 1.940 \\
Percentage of total variance & 36.222 & 62.049 \\
Number of test measures & 4 & 3 \\
\hline
\end{tabular}

A scree plot yielded a two-factor solution. Loadings $>0.40$ are shown in bold. 


\section{Cognitive basis of superior retention in the intentional group}

Participants' post-experimental reports suggested that the majority of participants (in both encoding groups) used an episodic-like means of recall (e.g., mentally reexperiencing the $W W W$ experience) when retrieving target $W W W$ information. This resonates with (1) previous research suggesting an important role of episodic memory in $W W W$ memory tasks (Holland and Smulders 2011; Easton et al. 2012; Cheke and Clayton 2013, 2015) and (2) the criteria of Pause et al. (2013) for an ecologically valid episodic memory task, which our $W W W$ task fulfills (see Introduction). Therefore, it is likely that the memory benefit of intentional encoding was due to the promotion of functions associated with the episodic memory system, though we acknowledge we cannot rule out the promotion of other cognitive functions that may have supported memory.

Given that our (intentional vs. incidental) encoding instructions came prior to the learning phase of the $W W W$ task, it is most likely that it was the encoding of novel episodic (target and contextual) information which was directly affected. This resonates with previous work showing that intentionally encoding novel information supports subsequent remembering (Craik and Tulving 1975; Neill et al. 1990; Holland and Smulders 2011). Specifically how was encoding affected? Given that participants in the intentional group were aware that their memory would be probed at a delayed stage, it is possible that encoding was supported by verbal mnemonic strategies (e.g., active rehearsal of item-location-session information). However, this is unlikely as all participants performed articulatory suppression (Hanley 1997) (repetition of "the, the, the...") during the encoding phase. We note that this does not eliminate the encoding and memorization of verbal information per se, but rather, reduces the likelihood of verbal strategies. Indeed, given the multisensory nature of episodic memory, it is likely that a combination of auditory/verbal, visual, and spatial information was encoded during the $W W W$ experience. A more plausible explanation is that, as a consequence of preexperimental instructions, participants in the intentional group demonstrated a general increase in perception/attention during coin hiding. It is possible that such an increase in perception/attention supported the encoding of novel sensory information relating to task-relevant target (item-location) information but also wider contextual information (e.g., the presence of environmental stimuli). Indeed, we found a similar memory benefit of intentional encoding across our measures of target ( $W W W$ task) and contextual (UCQs, WintR recall, and WintR recognition tests) information, suggesting that this was the case.

\section{Intentionality and relationships with episodic and nonepisodic factors}

In addition to the above, we aimed to explore specifically how encoding intentionality affected memory for target and contextual information by probing relationships between these measures and standard cognitive tests, which may uncover common underlying cognitive functions. Previous research demonstrates difficulty in establishing relationships between performances in different putative measures of episodic memory, possibly due to these different measures requiring the recruitment of different episodic (and possibly nonepisodic) cognitive function(s) (Holland and Smulders 2011; Cheke and Clayton 2013, 2015). Therefore, rather than attempting to establish direct relationships between measures of episodic memory and our memory measures, we extracted common factors of episodic and nonepisodic cognition from a set of standard cognitive tests. We then examined (1) whether these factors related to the retention of target and con- textual information and (2) whether intentionality of encoding affected these relationships.

As predicted, our results revealed that memory for target and contextual information was not related to the two nonepisodic cognitive functions that we tested (visual-spatial and verbal working memory) (Tulving 1972; Baddeley 2014). We cannot rule out the involvement of other nonepisodic cognitive functions, e.g., decision-making and planning, which were not probed.

In addition to the two nonepisodic factors, we extracted a total of four common factors of episodic cognition: (1) verbal memorization, (2) internal imagery, (3) mental manipulation of space, and (4) visual-spatial memory. The finding of multiple factors could indicate that the standard episodic memory tests performed by participants in our study involved at least four common underlying episodic cognitive functions, where each factor represents a specific episodic cognitive function. No relationships were found between memory for target $(W W W$ ) information and any of the factors of episodic cognition. However, relationships were observed between memory for contextual information (UCQs and WintR recall tests) and some of the factors of episodic cognition. Crucially, these relationships differed between the intentional and incidental groups, suggesting an effect of encoding intentionality (discussed shortly).

Why was memory for target $W W W$ information not related to any of the factors of episodic cognition, yet memory for some contextual information was? Assuming that the factors of episodic cognition each reflect specific cognitive functions, one possible explanation is that these factors simply did not reflect the cognitive functions recruited when performing the $W W W$ task (e.g., Plancher et al. 2008; Cheke and Clayton 2013), but did reflect the functions recruited in the UCQs and WintR recall tests. It is also possible that the multi-component nature of the $W W W$ task contributed to this null finding. Extracted factors of cognition were derived from direct measures of memory (e.g., total number of words recalled). However, performance in our $W W W$ task was measured via the combination of correctly recalled item, location, and session information, which will have induced more variability into the measure. Unlike the $W W W$ task, our UCQs and WintR recall and recognition measures provided a more direct measure of memory for contextual episodic information (e.g., total number of WintR words recalled). This may account, at least somewhat, for the relationships between these measures and the factors of episodic cognition, but not for the $W W W$ task.

Which factors of episodic cognition were related to memory for contextual information? Across both the intentional and incidental groups a significant positive correlation was observed between the WintR recall test (i.e., memory for contextual information-visually presented words-from the $W W W$ environment) and factor visual-spatial memory. Given that factor visual-spatial memory was derived from measures requiring the free recall of visual-spatial information, the positive correlations between this factor and performance in the WintR recall test is likely to reflect the recruitment of similar visual-spatial memory functions in these free recall measures. Despite a significant correlation between the free recall and recognition of WintR stimuli in the incidental group, no effect of factor visual-spatial memory was observed on the recognition of WintR stimuli. This is likely to reflect recognition memory drawing on different cognitive functions to those reflected by this "free recall" factor.

A significant positive correlation was also observed between the free recall of WintR stimuli-visually printed words-and factor verbal memorization in the intentional group. This is likely to be the result of our preexperimental encoding instructions. Participants in the intentional group were informed that their 
memory for target $W W W$ information would be probed later in the experiment. Participants in the incidental group were unaware that their memory would be probed subsequently. As discussed above, this manipulation is likely to have resulted in increased perception/attention that supported the encoding of target information and surrounding contextual information. It is possible that this resulted in the reading and thus encoding of WintR stimuli. Indeed, as previously noted, articulatory suppression during the $W W W$ encoding sessions would not necessarily eliminate the encoding of verbal information per se. No significant correlation was observed between verbal memorization and the free recall of WintR stimuli for participants in the incidental group, who are unlikely to have attended, read, and thus encoded WintR stimuli verbally. Rather, this information was likely encoded as visual-spatial information, as reflected by the correlation between factor visual-spatial memory and WintR recall test performance, discussed above. We note that free recall and recognition memory for WintR stimuli was generally poor across groups, and that a number of participants performed at floor levels. Thus, a lack of test sensitivity may have, at least somewhat, masked the presence/strength of correlations between derived factors of cognition and the WintR recall and recognition tests.

A significant positive correlation was found between memory for the presence/absence of environmental stimuli, as probed via the UCQs contextual memory measure, and factor mental manipulation of space for the intentional, but not incidental group. This suggests that, in the intentional group, participants who were better able to mentally manipulate a spatial framework were better at recalling wider contextual information from the $W W W$ task. Given that superior perception/attention for target and contextual information may have resulted in a deeper encoding and integration of novel information in the intentional group, we hypothesize that participants in this group formed a more unitary and accurate mental representation for the $W W W$ experience. An integrative representation would provide a framework for the mental reexperiencing, manipulation, inspection and extraction of target and contextual information relating to the $W W W$ experience. This could explain why participants in the intentional group demonstrated superior memory for contextual information and the relationship between participants' ability to mentally manipulate space and memory for contextual information. In contrast, we hypothesize that participants in the incidental group formed a less accurate and more fragmented representation of the $W W W$ experience. Such a representation would be less conducive for the mental reexperiencing and manipulation of the initial experience, and thus general memory performance.

This mental representation hypothesis could be evaluated in future work by examining relational memory for the $W W W$ experience. If intentional encoding supports the formation of an accurate and unitary mental representation, not only should memory for specific information (e.g., objects, locations, or object-location combinations) be enhanced (as evidenced in the current study), but, relational memory (e.g., spatial relationships between objects within the environment that were never directly experienced together) should also be superior. This could be examined via a delayed memory test probing the accuracy of a "cognitive map" (Tolman 1948), i.e., spatial relationships between objects in the environment, for the $W W W$ experience. Indeed, such tests provide a robust and sensitive means of the accuracy of the spatial framework of a mental representation for a recently experienced (virtual) environment (Weisberg et al. 2014; Craig et al. 2016).

We note that despite finding a number of significant interactions between group and factors of episodic cognition, many individual correlations did not reach significance. Furthermore, no consistent patterns were observed across our different measures. While we cannot completely rule out the possibility that this can be accounted for, at least somewhat, by noise in our data, this variation may be the result of the complex nature of episodic memory. Individual differences in the cognitive functions recruited when retrieving episodic memories are also likely to have contributed, at least somewhat, toward this inconsistency. Indeed, it is often assumed that putative episodic memory tests are solved via a similar method across individuals, which may not be strictly true, especially for more complex ecological measures such as the $W W W$ task.

\section{Summary}

Our results demonstrate that intentionally encoding a novel experience enhances the retention of target and wider contextual information. We found no relationships between memory for target and contextual information and factors of nonepisodic cognition, irrespective of encoding condition. However, we did find relationships between some of our contextual memory measures and factors of episodic cognition, and crucially, these relationships differed depending on whether this information was intentionally or incidentally encoded. Memory for contextual information was related to visual-spatial memory functions in both encoding groups, and to the mental manipulation of space and verbal memorization of material only in the intentional encoding group. We propose that these relationships reflect the recruitment of differing underlying episodic cognitive functions and can be explained by our intentionality manipulation. Specifically, we propose that intentional encoding led to superior perception/attention, which supported the encoding/formation of a more unitary and accurate mental representation of the initial experience. This will have supported subsequent mental reexperiencing, internal manipulation, and extraction of target and contextual information during delayed testing. In contrast, participants who incidentally encoded the initial experience will have formed a less accurate and more fragmented representation, hampering subsequent mental reexperiencing and general memory performance. This hypothesis to explain our results remains to be tested. Further characterization of the effect of encoding intentionality in memory is therefore still required.

\section{Materials and Methods}

\section{Ethics statement}

This research was approved by Newcastle University's Faculty of Medical Sciences Research Ethics Committee (Ref: 00414). All participants provided their informed consent in writing prior to participating.

\section{Participants}

Forty-nine healthy adults (mean age $=29.71, S D=12.45$ ) who were fluent in the English language were recruited as participants. Participants were assigned to one of two between-subjects $W W W$ task groups: (1) incidental encoding $(N=25$; 13 males, 12 females; $M=27.3 \mathrm{yr}, S D=1.92)$ and (2) intentional encoding $(N=24 ; 10$ males, 14 females; $M=32.3 \mathrm{yr}, S D=2.99)$. Participants were allocated to groups at the experimenter's discretion to balance groups for sex and age as best possible. Groups did not differ significantly in terms of age $\left(F_{(1,47)}=1.992, P=0.165\right)$ or sex $\left(\chi^{2}=1.311, P=0.252\right)$.

\section{Design}

Participants performed a number of tests of episodic and nonepisodic cognition. A between-subject design was adopted for the $W W W$ task, UCQs and WintR tests with between-subjects factor 
intentionality of encoding (intentional vs. incidental groups). Performance of all other tasks was identical for all participants, i.e., there was no between-subject manipulation of intentionality of encoding.

\section{Procedure}

Figure 4 provides an overview of the procedure. To avoid an excessive duration, the procedure was designed in such a way that cognitive tests were embedded within one another. The order of test performance was identical for all participants. Participants began by performing the first of two $W W W$ task encoding sessions, followed by a visual-spatial memory task. A 2-h break then followed during which the participant left the lab and was free to go about their daily activities. Following the break, participants performed the second of two $W W W$ task encoding sessions, followed by a number of common episodic and nonepisodic cognitive tests with a total duration of $2 \mathrm{~h}$. Finally, participants were asked to recall information pertaining to the earlier $W W W$ task encoding sessions. All cognitive tests are detailed below.

\section{What-Where-When task}

WWW task environment. The $W W W$ task was performed in an unoccupied, cluttered office $(4.6 \mathrm{~m} \times 3.9 \mathrm{~m})$ within the Institute of Neuroscience, Newcastle University. Figure 5 provides an illustration of this space and the arrangement of selected everyday items within the room.

WWW task preexperimental instructions (between-subject manipulation). The critical between-subjects manipulation in the $W W W$ task was the intentionality of encoding (intentional vs. incidental). This manipulation was implemented via the preexperimental instructions given to the participant by the experimenter.

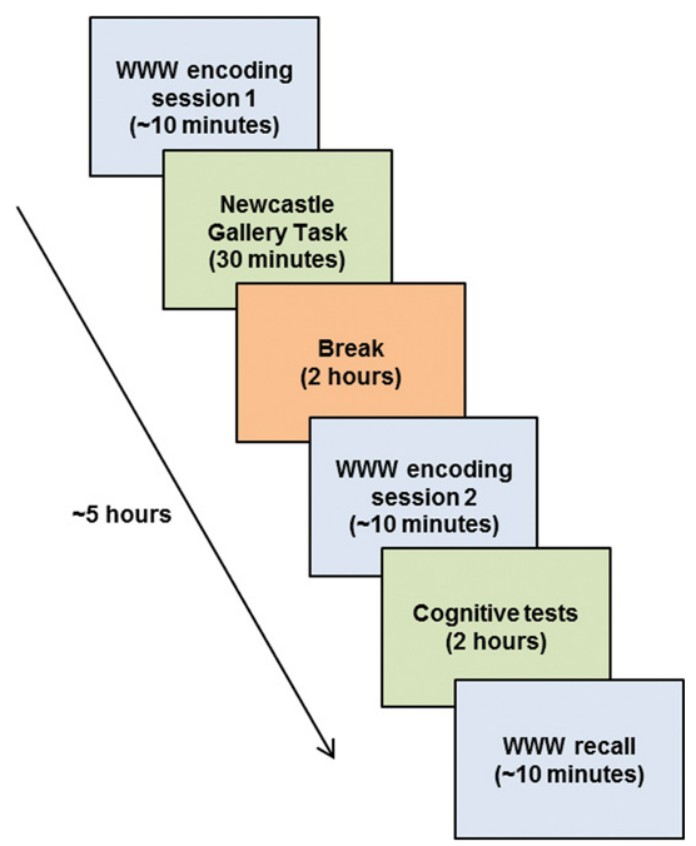

Figure 4. Procedure overview. Participants performed a number of different cognitive tests that examined episodic and nonepisodic cognition. The $W W W$ task spanned the overall procedure. Two $W W W$ encoding sessions took place, separated by the performance of a visual-spatial longterm memory test (Newcastle gallery task), and a 2-h break. The second WWW encoding session was followed by the performance of a number of common episodic and nonepisodic memory tests lasting 2 $\mathrm{h}$. The recall of $W W W$ information from both encoding sessions occurred at the end of the procedure. All participants performed tests in the same order with a total duration of $\sim 5 \mathrm{~h}$.
For the intentional group, the experimenter instructed participants that they would be required to hide several items within a cluttered office in two sessions. Importantly, they were also informed that later in the procedure they would be required to recall which item they had hidden (what), in which location (where). To ensure that participants in the intentional group were motivated, prior to encoding session one, they were instructed that they would receive a performance-based reward for the subsequent recall of correct $W W W$ information. Finally, participants in the intentional group were instructed that they would be required to repeat aloud "the, the the..." while hiding items around the cluttered room. This was a means of including articulatory suppression during encoding to reduce the likelihood of verbal coding and active rehearsal of $W W W$ (item-location-session) information.

For the incidental group, the experimenter provided participants with a cover story that we were examining the possible effects that performing a motor task (coin hiding) has on the phonological loop (repetition of "the, the the..."). This cover story enabled the inclusion of articulatory suppression, as in the intentional group. Participants in the incidental group were not informed that they would perform a subsequent memory test for $W W W$ (item-location-session) information, or that they would receive a performance-based reward for the correct recall of this information.

WWW task encoding phase. Two WWW encoding sessions occurred during the procedure (see Fig. 4). At the start of both encoding sessions, the experimenter handed the participant eight "to-be-hidden" coins: four of high value (20 pence) and four of low value ( 2 pence). Participants in both groups were instructed that they would be required to hide the eight coins in eight different predetermined locations around the room. Participants were notified of locations on a sequential basis by the experimenter who walked around the room silently pointing at each location where a coin should be hidden. Participants were free to choose the coin (from the selection in hand) to be hidden in each location. The locations used, and sequence of locations, was identical for all (incidental and intentional group) participants. In total, participants hid a total of 16 coins $(8 \times 2$ pence and $8 \times 20$ pence), in 16 different, predetermined locations, over two encoding sessions. This provided our critical measure, the retention of coin (what), location (where), and session (when) information.

WWW task recall phase. The recall phase of the $W W W$ task was identical for both encoding groups. All participants were returned to the $W W W$ task environment and asked to recall $W W W$ information, i.e., which coin type (what) they had hidden in which location (where), during which encoding session (when). All participants were instructed that they would receive a monetary performance-based reward of the cumulative value of the coin type (what) from the first five correctly recalled $W W W$ combinations, e.g., if the first five correctly recalled $W W W$ combinations all included a 20 pence coin as the what component, they would receive a total of $£ 1$. These instructions acted as a reminder for participants in the intentional group, whereas this was the first time the incidental group learned of this performance-based reward.

When participants were unable to recall any further $W W W$ information, they were asked: "How did you recall the information of which coin you had hidden, in which location, and in which hiding session?" In asking this question we aimed to establish whether the participant used episodic-like recall to solve the $W W W$ task. We classified participant responses as: (1) "remember," i.e., they used episodic-like recall or (2) "know," i.e., they did not use an episodic-like recall. The "know" response was perceived as using semantic memory to solve the $W W W$ task (Gardiner et al. 2006). If an unclear/ambiguous response was received, we further probed the participant, asking them whether they "remembered" their earlier movements during the encoding sessions, or whether they simply "knew" the type of coins hidden 
The What-Where-When (WWW) task environment

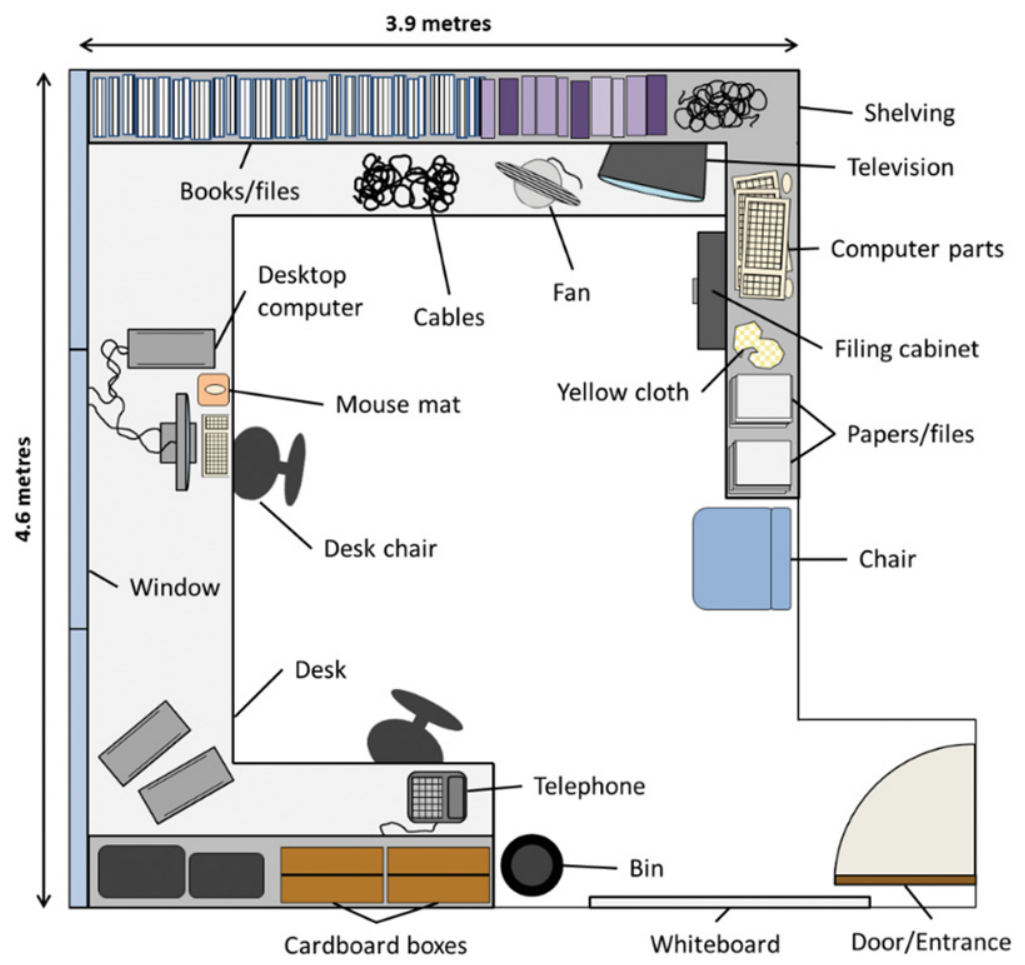

Figure 5. The What-Where-When (WWW) task environment. An illustration of the layout of the environment where the $W W W$ task took place. The room was an unoccupied, cluttered office within the Institute of Neuroscience, Newcastle University. Over two encoding sessions, 16 coins $(8 \times 20 p$ and $8 \times 2 p$ ) were hidden in 16 predetermined locations within the $W W W$ task environment.

in different locations. Thus, a remember/know distinction was obtained for all participants. Participants' descriptions that were initially categorized as being "episodic-like" were further categorized on a post hoc basis depending on the predominant strategy reported by the participant. The vast majority of these descriptions fell into two categories: (1) mentally reliving/reexperiencing the $W W W$ experience or (2) using items within the environment as cues to recall information from the initial experience.

$W W W$ task data were transformed into proportions via the following rules. For overall $W W W$ task performance, the number of correctly recalled $W W W$ combinations (i.e., when all coin-what, location-where, session-when components were correct) was divided by the total number of $W W W$ combinations, i.e., 16 (e.g., $5 / 16=0.313$ ). To achieve a normal distribution, proportions were transformed to arcsine square root values for analyses.

\section{Measures of contextual memory from the WWW experience}

The $W W W$ task environment provided opportunity to include further measures of incidental encoding and memory for contextual features, i.e., the presence/absence of objects in the $W W W$ environment (see Fig. 5 for examples of items in the environment). These measures comprised: (1) a set of unexpected context questions (UCQs) pertaining to contextual features of the $W W W$ task environment during encoding sessions one and two and (2) a "words in the room" (WintR) test which was also unexpected and examined memory for words placed in salient locations around the $W W W$ task environment during encoding sessions 1 and 2. Crucially, participants in neither encoding group were informed that their memory would be subsequently probed for this contextual information.
Unexpected context questions. Prior to performing the $W W W$ task recall session, all participants were unexpectedly asked 20 questions regarding the context of the $W W W$ task environment, e.g., "Earlier in the procedure, can you remember whether the window blinds were lowered in the first coin hiding session and/or the second coin hiding session?" Participants were required to provide two responses for each of the 20 questions: one response pertaining to the environmental context of $W W W$ encoding session 1, and a second response pertaining to the environmental context of $W W W$ encoding session 2. Therefore, 40 responses were collected in total $(20 \times$ encoding session $1,20 \times$ encoding session 2 ). There was an equal number of questions that were (1) true for both $W W W$ encoding sessions, (2) false for both $W W W$ encoding sessions, (3) true only for $W W W$ encoding session 1, and (4) true only for $W W W$ encoding session 2. All questions probed contextual memory for information that was not essential to solving the $W W W$ task, i.e., questions probed memory for objects that were not used as coin-hiding locations. The experimenter verbally presented all questions, with participants required to provide verbal responses, stating the $W W W$ encoding sessions (one, two, both, neither) that they deemed the question to be true for. The total number of correct responses was divided by 40 (total number of questions) to provide a proportion correct score. To achieve a normal distribution, these values were transformed to arcsine square root values for analyses.

Words in the room. Similar to the UCQs, the Words in the Room (WintR) test came as a surprise. Two WintR test measures were used: (1) a free recall of WintR words and (2) a recognition test for WintR words. Twenty-eight words from the RAVLT (Geffen et al. 1990) word recall test were selected. Words were presented as large, bold black text on white A4 paper and were placed in various salient locations around the $W W W$ task environment. As for the UCQs, none of these locations were used in the $W W W$ task as coin-hiding locations. The 28 word stimuli were divided equally into four groups: (1) seven words were presented in $W W W$ encoding session 1 only, (2) seven words presented in $W W W$ encoding session 2 only, (3) seven words presented in $W W W$ encoding sessions 1 and 2 , and (4) seven words not presented in either $W W W$ encoding session one or two (foils). Following performance of the UCQs, participants were asked to freely recall as many of the WintR words that were presented during the two $W W W$ encoding sessions. The proportion of correct words recalled was calculated and these values were transformed to arcsine square root values for analyses.

Participants also performed a recognition test for WintR stimuli. In this recognition test, participants were presented the 28 WintR words (21 targets and seven foils) in large, bold, black text on a white background on a computer screen, i.e., in a similar visual form as during the $W W W$ task encoding sessions. Participants were required to verbally state the $W W W$ encoding sessions that they believed each word was present in. Participants were asked to provide a response in the form of (1) $W W W$ encoding session one only, (2) $W W W$ encoding session two only, (3) both $W W W$ encoding sessions, or (4) neither $W W W$ encoding sessions. Responses were required to be correct 
for both encoding sessions to be scored as correct. For example, if a word appeared in session one only and the participant responded with "both sessions," this would be scored as incorrect. In contrast, if the participant responded with "session one only," this would be scored as correct. The total number of correct responses was divided by 28 (total number of words; 21 targets and seven foils) to provide a proportion correct score. These values were transformed to arcsine square root values for analyses.

\section{Other cognitive measures}

To examine whether memory for target and contextual information from the $W W W$ experience was related to performance in standard measures of episodic and nonepisodic cognition, we selected a number of common episodic and nonepisodic (working memory) cognitive measures. Measures of episodic memory functions included: visual-spatial memory (Burgess et al. 2002; Plancher et al. 2012), visual imagery (Szpunar et al. 2007; de Borst et al. 2012), and the internal manipulation of visual imagery (Perner et al. 2010; Rice and Rubin 2011). Measures of working memory functions included: verbal and visual-spatial working memory tests (Baddeley 2014).

\section{Measures of episodic cognition}

Wordlist learning. Participants were orally presented a prerecorded list of 30 high-frequency nouns and immediately asked to recall them. The list comprised an equal number of positive, negative, and neutral words (Pariante et al. 2012). Following a filled 30-min delay, during which participants performed other, unrelated cognitive tests, participants were again asked to recall the word list. This provided us with both immediate and delayed recall measures. The number of words recalled during the delayed recall test was used for analysis as this reflected long-term (episodic) memory. For the free recall test, the total number of words recalled at the delayed word recall test performance was extracted.

Object relocation task. In the Object Relocation task (Kessels et al. 1999), participants were presented five subtests on a touchscreen computer: object memory (OM), visual spatial reconstruction (VSR), positional object memory (POM), object location binding (OLB), and combined object memory (COM). Each subtest (other than the VSR) comprised two trials: (1) immediate recall-where there was no delay between learning and test and (2) delayed recall-where there was a 3-min retention delay (filled with other, unrelated cognitive tests, e.g., digit span, verbal fluency) between learning and test. As we were interested in the long-term retention of episodic information, delayed recall trials were used in subsequent analyses as working memory functions could not account for memory retention due to the 3-min retention delay being filled with other unrelated cognitive tasks, thus blocking the online maintenance of information (e.g., active verbal rehearsal).

In the OM subtest, participants were required to remember 10 different everyday items (e.g., telephone, car) that were presented onscreen for $20 \mathrm{sec}$. During test, participants were presented the 10 objects that were presented during learning, alongside 10 never-experienced objects. Using the touchscreen computer, participants were required to select the 10 objects that had been earlier presented. The percentage of incorrectly identified items was extracted and converted to a percentage correct score for analyses.

In the VSR subtest, participants were required to recreate the spatial layout of an array of everyday items. Ten different items were presented in a box on the left of the screen. On the right of the screen was an unfilled box, in which, using the touchscreen, participants were required to replicate the spatial layout of items shown on the left. The total distance of error $(\mathrm{mm})$ between actual and correct positions for all items was extracted for analyses and subtracted from the POM and COM measures to control for individual differences in spatial reconstruction.
In the POM test, participants were required to remember the locations of 10 identical everyday items that were presented onscreen for $20 \mathrm{sec}$. During test, using the touchscreen, participants were required to recreate the spatial arrangement of items by placing them in their earlier held locations. The total distance of error $(\mathrm{mm})$ between the location of objects in the reconstructed array and the nearest correct location (i.e., "best-fit" error, $\mathrm{mm}$ ) was extracted. For analyses, these values were reversed as a function of the single largest error score $(\mathrm{mm})$ collected from all participants (largest error - error score $=$ reversed score) so that larger values reflected greater performance (accuracy).

In the OLB test, participants were required to remember the locations of 10 different everyday items that were presented onscreen for $20 \mathrm{sec}$. During test, using the touchscreen, participants were required to place items in their earlier held locations. All locations where an item was presented were identified via a black circle. The percentage of items placed in the incorrect locations was extracted and converted to a percentage correct score for analyses.

In the COM test, participants were required to remember the locations of 10 different everyday items that were presented onscreen for $20 \mathrm{sec}$. During test, using the touchscreen, participants were required to place items in their earlier held locations. The mean distance of error $(\mathrm{mm})$ between the correct locations of items and the actual locations of items (in the reconstructed array during test) was extracted for analyses.

Visual imagery task. In the visual imagery task (Hanley et al. 1991), participants' ability to internally generate visual imagery was probed. Participants were presented three questions on a touchscreen computer, each comprising 16-20 trials. The three questions were (1) Which is the larger of these two objects? (2) Which of these two animals has a long/short tail? (3) Typically which is the larger of these two animals? In each trial, the participant was presented with two possible responses on the touch screen, one correct and one incorrect (e.g., question 1: "chair or house"; question 2: "rabbit or lion"; question 3: "mouse or horse"). Participants responded via selecting the correct response on the touchscreen. The total number of correct responses (over the three questions, 52 trials in total) was extracted for analyses.

Northumberland Gallery Task. The Northumberland gallery task (NGT) examined participants egocentric and allocentric visual-spatial memory (Nilsson et al. 2013). Participants were first trained using a 3D cardboard model until they were familiar with the environment before performing the computerized NGT task. Participants were presented a circular virtual environment onscreen-their vantage point within the environment was from a raised viewing platform above the walls of the circular environment. On the inner (stone texture) walls of the environment were seven "paintings" of different animals that acted as environmental reference points. Trials were broken down into learning and test phases. In the learning phase, a tall green cylinder was presented at a predetermined location on the (wooden texture) floor. The screen then went blank and the participant was provided with instructions onscreen of the type of test phase they would face. There were three possible test phases: (1) where the vantage point (on the raised platform) within the environment was manipulated-allocentric visualspatial memory, (2) where the vantage point within the environment remained the same, but the walls of the environment had rotated-egocentric visual-spatial memory, and (3) where no manipulations occurred-control. In each test type, participants were presented two small dots on the floor of the environment (one white, one orange) and were required to respond (by pressing corresponding keyboard keys) with the correct location of the earlier presented green cylinder. The mean percentage of correct trials for the allocentric (NGTa) and egocentric (NGTe) visual-spatial memory measures were extracted for analyses. All participants performed at, or near, ceiling in the control condition. 
Mental rotation test. In the mental rotation task (MRT) (Vandenberg and Kuse 1978), participants were presented with two shapes comprising numerous cubes on a touchscreen computer. Participants were required to determine whether the two shapes were identical, but rotated versions of one another or different shapes by providing a "same/different" response on the touchscreen. The total number of correct responses was extracted for analyses.

\section{Measures of nonepisodic cognition}

Visual patterns test. In the computerized visual patterns test (VPT) (Della Sala et al. 1999), participants were presented a part-filled grid on a touchscreen computer. Following a 3-sec presentation, participants were presented the same grid, now unfilled. Using the touchscreen, participants were required to replicate the earlier presented part-filled grid by touching the previously filled squares. As the VPT progressed, test demand (i.e., the complexity and size of to-be-retained patterns) increased. The maximum number of filled squares in a presented pattern was extracted as span for analyses.

Newcastle Spatial Working Memory Test. In the computerized Newcastle spatial working memory (NSWM) test (Pariante et al. 2012), participants were presented an array of "bins" (colored shapes), under which a set number of tokens were hidden. Participants were required to search the different bins (via mouse clicks) for hidden tokens. The participant was required to search bins until all tokens were found. As the task progressed, the number of bins in an array, and the number of to-be-found tokens increased, thus increasing task demand. Two measures were extracted for analyses: between errors (revisiting bins which have already been found to contain a token in a previous search sequence) and within errors (revisiting empty bins within the same search sequence).

CANTAB spatial span. In the computerized CANTAB spatial span test (Cambridge-Cognition, 2006), participants were presented an array of unfilled boxes on a touch-screen computer. In each trial, a number of boxes would be filled in a sequential order. Following completion of the sequence, the participant was required to repeat the sequence by pressing the correct boxes on the touchscreen. As the task progressed, the number of filled boxes in a sequence increased until the participant was unable to recall the correct sequence of boxes. The sequence containing the maximum number of boxes that a participant was able to correctly recall was extracted as span for analyses.

Digit span test. Participants performed both a forward and reverse verbal digit span test (Spreen and Strauss 1998). Participants were verbally presented a sequence of digits by the experimenter at a pace of one per second, after which they were required to verbally recall to the experimenter. In the forward digit span test, participants were required to recall digits in the same order as presentation. In the reverse digit span test, participants were required to recall digits in the reverse order. As both tests progressed, cognitive demand, i.e., the number of to-be-retained digits in a sequence, increased. The longest sequence of digits correctly recalled was extracted from the forward and reverse tests as span for analyses.

Verbal fluency test. In the verbal fluency test (Spreen and Strauss 1998), participants performed three trials. In each trial, participants were presented one letter from the English alphabet (F, A, or S). Following presentation of a letter (e.g., F), participants were required to verbally respond with as many words as possible beginning with the corresponding letter over a 60 -sec period. Any repetitions, plurals of already stated words, or names of people or places were not counted. The total number of valid words stated during the three (F, A, and S) trials was recorded for analyses.

\section{Statistical analyses}

The $\alpha$ level was set to 0.05 for all analyses. Univariate ANOVAs were used to examine the effects of our experimental manipulation (intentionality of encoding) on memory for target ( $W W W$ task) and contextual (UCQs, WintR recall and WintR recognition tests) information. Pearson correlation coefficients were calculated to examine the relationships between performances in the different measures of target and contextual memory. Correlation coefficients were also transformed via Fisher r-to-z transformation to enable their comparison between groups. To examine how memory for target and contextual information related to common factors of episodic and/or nonepisodic cognition, principal component analyses (PCA) with oblique Promax rotations were performed to reduce the data from many episodic and nonepisodic measures to a smaller number of variables, which is the recommended method when correlations are likely (Abdi 2003; Costello and Osborne 2005; Brown 2009). The relationships between the generated PCA factors and memory for target and contextual information were examined by conducting Univariate ANCOVAs in which the $W W W$ task, UCQs, WintR recall, and WintR recognition measures were independently used as the dependent variable; group was used as a fixed factor, and the four episodic-like PCA factors were used as covariates. Models included interactions between group and each episodic-like PCA factor. A modelsimplification approach (Crawley 2014) was adopted where the most nonsignificant interaction was removed. If the model did not produce any significant findings, the main effect associated with the removed interaction was also removed from the model. This process continued in a stepwise manner until significant findings were observed or all main effects and interactions were removed from the model.

\section{Acknowledgments}

The authors thank all participants who gave their time to take part in our research. We also thank two anonymous reviewers for their helpful comments that enabled us to improve our manuscript. P.G. received Research Capability Funding from the Northumberland, Tyne and Wear NHS Foundation Trust.

\section{References}

Abdi H. 2003. Factor rotations in factor analyses. In Encyclopedia of social sciences research methods, (ed. Bryman A, Futing T), pp. 1-8. Sage, Thousand Oaks, CA.

Addis DR, Pan L, Vu M-A, Laiser N, Schacter DL. 2009. Constructive episodic simulation of the future and the past: Distinct subsystems of a core brain network mediate imagining and remembering. Neuropsychologia 47: 2222-2238.

Babb SJ, Crystal JD. 2005. Discrimination of what, when, and where: Implications for episodic-like memory in rats. Learn Motiv 36: 177-189.

Baddeley A. 2014. Working memory. Science, New Series 255: 556-559.

Berntsen D, Staugaard S, Sørensen L. 2013. Why am I remembering this now? Predicting the occurrence of involuntary (spontaneous) episodic memories. J Exp Psychol Gen 142: 426-444.

Brown JD. 2009. Choosing the right type of rotation in PCA and EFA. Shiken JALT Test Eval SIG Newsl 13: 20-25.

Burgess N, Maguire EA, O'Keefe J. 2002. The human hippocampus and spatial and episodic memory. Neuron 35: 625-641.

Cambridge Cognition. 2006. Neuropsychological test automated battery (CANTABeclipse) manual. Cambridge Cognition Limited, Cambridge, UK.

Cheke LG, Clayton NS. 2013. Do different tests of episodic memory produce consistent results in human adults? Learn Mem 20: 491-498.

Cheke LG, Clayton NS. 2015. The six blind men and the elephant: Are episodic memory tasks tests of different things or different tests of the same thing? J Exp Child Psychol 137: 164-171.

Clayton NS, Dickinson A. 1998. Episodic-like memory during cache recovery by scrub jays. Nature 395: $4-6$.

Costello AB, Osborne JW. 2005. Best practices in exploratory factor analysis: four recommendations for getting the most from your analysis. Pract Assessment Res Eval 10: 1-9.

Craig M, Dewar M, Harris MA, Della Sala S, Wolbers T. 2016. Wakeful rest promotes the integration of spatial memories into accurate cognitive maps. Hippocampus 26: 185-193. 
Craik FIM, Tulving E. 1975. Depth of processing and the retention of words in episodic memory. J Exp Psychol Gen 104: 268-294.

Crawley MJ. 2014. Statistics: an introduction using R. 2nd Ed. Wiley-Blackwell, Chichester.

de Borst AW, Sack AT, Jansma BM, Esposito F, de Martino F, Valente G, Roebroeck A, di Salle F, Goebel R, Formisano E. 2012. Integration of "what" and "where" in frontal cortex during visual imagery of scenes. Neuroimage 60: $47-58$.

Della Sala S, Gray C, Baddeley A, Allamano N, Wilson L. 1999. Pattern span: A tool for unwelding visuo-spatial memory. Neuropsychologia 37: $1189-1199$.

Easton A, Webster LAD, Eacott MJ. 2012. The episodic nature of episodic-like memories. Learn Mem 19: 146-150.

Eichenbaum H. 2004. Hippocampus: Cognitive processes and neural representations that underlie declarative memory. Neuron 44: $109-120$.

Gardiner JM, Gregg VH, Karayianni I. 2006. Recognition memory and awareness: Occurrence of perceptual effects in remembering or in knowing depends on conscious resources at encoding, but not at retrieval. Mem Cognit 34: 227-239.

Gavett BE, Horwitz JE. 2012. Immediate list recall as a measure of short-term episodic memory: Insights from the serial position effect and item response theory. Arch Clin Neuropsychol 27: 125-135.

Geffen G, Moar KJ, O'hanlon AP, Clark CR, Geffen LB. 1990. Performance measures of 16- to 86-year-old males and females on the auditory verbal learning test. Clin Neuropsychol 4: 45-63.

Grady CL, McIntosh AR, Rajah MN, Craik FI. 1998. Neural correlates of the episodic encoding of pictures and words. Proc Natl Acad Sci 95: $2703-2708$.

Hanley JR. 1997. Does articulatory suppression remove the irrelevant speech effect? Memory 5: 423-431.

Hanley JR, Young AW, Pearson NA. 1991. Impairment of the visuo-spatial sketch pad. Q J Exp Psychol A 43: 101-125.

Hassabis D, Kumaran D, Vann SD, Maguire EA. 2007. Patients with hippocampal amnesia cannot imagine new experiences. Proc Natl Acad Sci 104: $1726-1731$.

Holland SM, Smulders TV. 2011. Do humans use episodic memory to solve a What-Where-When memory task? Anim Cogn 14: 95-102.

Inostroza M, Binder S, Born J. 2013. Sleep-dependency of episodic-like memory consolidation in rats. Behav Brain Res 237: 15-22.

Kessels RP, Postma A, de Haan EH. 1999. Object Relocation: A program for setting up, running, and analyzing experiments on memory for object locations. Behav Res Methods Instrum Comput 31: 423-428.

Mazurek A, Bhoopathy RM, Read JCA, Gallagher P, Smulders TV. 2015. Effects of age on a real-world what-where-when memory task. Front Aging Neurosci 7: 1-17.

Meulenbroek O, Petersson KM, Voermans N, Weber B, Fernández G. 2004. Age differences in neural correlates of route encoding and route recognition. Neuroimage 22: 1503-1514.

Neill WT, Beck JL, Bottalico KS, Molloy RD. 1990. Effects of intentional versus incidental learning on explicit and implicit tests of memory. J Exp Psychol Learn Mem Cogn 16: 457-463.

Nilsson J, Ferrier IN, Coventry K, Bester A, Finkelmeyer A. 2013. Negative BOLD response in the hippocampus during short-term spatial memory retrieval. J Cogn Neurosci 25: 1358-1371.
Pariante CM, Alhaj HA, Arulnathan VE, Gallagher P, Hanson A, Massey E, McAllister-Williams RH. 2012. Central glucocorticoid receptor-mediated effects of the antidepressant, citalopram, in humans: A study using EEG and cognitive testing. Psychoneuroendocrinology 37: 618-628.

Pause BM, Zlomuzica A, Kinugawa K, Mariani J, Pietrowsky R, Dere E. 2013. Perspectives on episodic-like and episodic memory. Front Behav Neurosci 7: 33 .

Pengas G, Patterson K, Arnold RJ, Bird CM, Burgess N, Nestor PJ. 2010. Lost and found?: bespoke memory testing for Alzheimer's disease and semantic dementia. 21: $1347-1365$

Perner J, Kloo D, Rohwer M. 2010. Retro- and prospection for mental time travel: Emergence of episodic remembering and mental rotation in 5- to 8-year old children. Conscious Cogn 19: 802-815.

Plancher G, Nicolas S, Piolino P. 2008. Virtual reality as a tool for assessing episodic memory. In Proceedings of the 2008 ACM symposium on virtual reality software and technology, pp. 179-182. ACM, New York, NY, USA.

Plancher G, Gyselinck V, Nicolas S, Piolino P. 2010. Age effect on components of episodic memory and feature binding: A virtual reality study. Neuropsychology 24: 379-390.

Plancher G, Tirard A, Gyselinck V, Nicolas S, Piolino P. 2012. Using virtual reality to characterize episodic memory profiles in amnestic mild cognitive impairment and Alzheimer's disease: Influence of active and passive encoding. Neuropsychologia 50: 592-602.

Rice HJ, Rubin DC. 2011. Remembering from any angle: The flexibility of visual perspective during retrieval. Conscious Cogn 20: 568-577.

Schacter DL, Addis DR, Buckner RL. 2007. Remembering the past to imagine the future: The prospective brain. Nat Rev Neurosci 8: 657-661.

Schacter DL, Addis DR, Hassabis D, Martin VC, Spreng RN, Szpunar KK. 2012. The future of memory: Remembering, imagining, and the brain. Neuron 76: 677-694.

Spreen O, Strauss E. 1998. Compendium of neuropsychological tests, administration, norms and commentary. Oxford University Press, Oxford.

Szpunar KK, Watson JM, McDermott KB. 2007. Neural substrates of envisioning the future. Proc Natl Acad Sci 104: 642-647.

Tolman EC. 1948. Cognitive maps in rats and men. Psychol Rev 55: $189-208$.

Tulving E. 1972. Episodic and semantic memory. In Organisation of memory (ed. Tulving E, Donaldson W), pp. 381-403, Academic Press, New York.

Tulving E. 1985. Elements of episodic memory. Oxford University Press, Oxford.

Vandenberg SG, Kuse AlR. 1978. Mental rotations, a group test of three-dimensional spatial visualization. Percept Mot Skills 47: 599-604.

Weisberg SM, Schinazi VR, Newcombe NS, Shipley TF, Epstein RA. 2014. Variations in cognitive maps: Understanding individual differences in navigation. J Exp Psychol Learn Mem Cogn 40: 669-682.

Zentall TR, Singer RA, Stagner JP. 2008. Episodic-like memory: Pigeons can report location pecked when unexpectedly asked. Behav Processes 79: 93-98.

Zinkivskay A, Nazir F, Smulders TV. 2009. What-where-when memory in magpies (Pica pica). Anim Cogn 12: 119-125.

Received December 22, 2015; accepted in revised form August 15, 2016. 


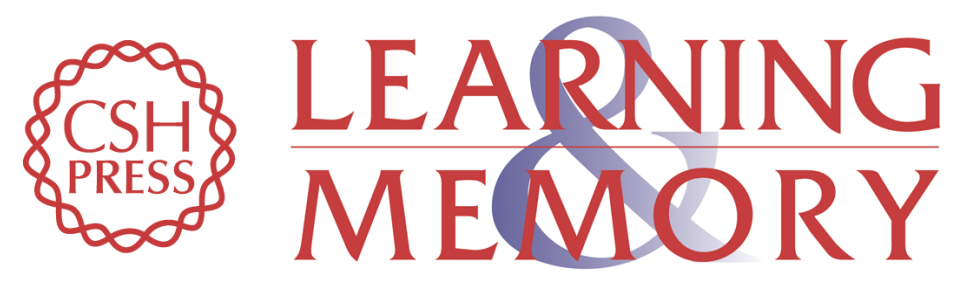

\section{How does intentionality of encoding affect memory for episodic information?}

Michael Craig, Karla Butterworth, Jonna Nilsson, et al.

Learn. Mem. 2016, 23:

Access the most recent version at doi:10.1101/Im.041491.115

References This article cites 44 articles, 5 of which can be accessed free at:

http://learnmem.cshlp.org/content/23/11/648.full.html\#ref-list-1

Creative This article is distributed exclusively by Cold Spring Harbor Laboratory Press for the

Commons

License

first 12 months after the full-issue publication date (see

http://learnmem.cshlp.org/site/misc/terms.xhtml). After 12 months, it is available under a Creative Commons License (Attribution-NonCommercial 4.0 International), as described at http://creativecommons.org/licenses/by-nc/4.0/.

Email Alerting Receive free email alerts when new articles cite this article - sign up in the box at the Service top right corner of the article or click here. 